\title{
On positive-realness and stability of switched linear differential systems
}

\author{
Jonathan C. Mayo-Maldonado, Paolo Rapisarda
}

\begin{abstract}
We present some results regarding the stability of switched linear differential systems (SLDS) in the behavioral framework. Positive-realness is studied as a sufficient condition for stability and some implications derived from the use of positive-real completions are discussed.

Index Terms-switched systems; behaviors; quadratic differential forms; positive-realness.
\end{abstract}

\section{INTRODUCTION}

A switched system is a set of dynamical systems with a rule that orchestrates the switching among them [2]. They are usually studied in the state space framework: all the dynamical regimes share the same state space, i.e. in the linear case each system is described by $\frac{d}{d t} x=A x+B u$; or in descriptor form $E \dot{x}=A x+B u$, where $E$ is a singular matrix, [11]. In [6],[5], a new approach has been put forward in which the dynamical regimes do not necessarily share the same state space, and they are described by sets of higherorder differential equations. We call these switched linear differential systems (SLDS).

Switching between stable systems may give rise to unstable responses (see [2], pp.19-20); consequently, it is important to find conditions that guarantee asymptotic stability (see e.g. [2],[3],[8]). In the state space setting, the notion of positive realness has been employed for the analysis and derivation of sufficient conditions of stability for switched linear systems (see e.g. [7],[14]). In the linear differential systems case, some results have been presented in [6],[5] using positive-realness as a sufficient condition for stability. In this contribution we present several new results using the the concept of positive-real completion.

\section{BACKGROUND}

\section{A. Notation}

The space of real vectors with $\mathrm{n}$ components is denoted by $\mathbb{R}^{n}$, and the space of $n \times m$ real matrices by $\mathbb{R}^{m \times n}$. The ring of polynomials with real coefficients in the indeterminate $\xi$ is denoted by $\mathbb{R}[\xi]$; the ring of two-variable polynomials with real coefficients in the indeterminates $\zeta$ and $\eta$ is denoted by $\mathbb{R}[\zeta, \eta] . \mathbb{R}^{\mathrm{n} \times \mathrm{m}}[\xi]$ is the space of $\mathrm{n} \times \mathrm{m}$ polynomial matrices in $\xi$, and the space of $\mathrm{n} \times \mathrm{m}$ polynomial matrices in $\zeta$ and $\eta$ is denoted by $\mathbb{R}^{\mathrm{n} \times \mathrm{m}}[\zeta, \eta]$. A polynomial $p \in \mathbb{R}[\xi]$ is Hurwitz if its roots are all in the open left half-plane.

We now introduce the concept of $R$-canonical representative of a polynomial differential operator. Given $R \in \mathbb{R}^{\mathrm{w} \times \mathrm{w}}[\xi]$ nonsingular, and $f \in \mathbb{R}^{1 \times \mathrm{w}}[\xi] ; f$ can be uniquely written

J.C. Mayo-Maldonado and P. Rapisarda are with the CSPC group, School of Electronics and Computer Science, University of Southampton, Great Britain, e-mail: $\{$ jcmm1g11, pr3\}@ecs . soton . ac . uk, Tel: $+(44) 2380593367$, Fax: +(44)2380594498. as $f R^{-1}=s+n$, where $s$ is a vector of strictly proper rational functions, and $n \in \mathbb{R}^{1 \times \mathrm{w}}[\xi]$. We define the (polynomial) $R$-canonical representative of $f$ as $(f \bmod R)(\xi):=$ $s(\xi) R(\xi)$. The definition of $R$-canonical representative is extended in a natural way to polynomial matrices.

The set of infinitely-differentiable functions from $\mathbb{R}$ to $\mathbb{R}^{\mathrm{w}}$ is denoted by $\mathfrak{C}^{\infty}\left(\mathbb{R}, \mathbb{R}^{\mathrm{w}}\right)$. Given $f: \mathbb{R} \rightarrow \mathbb{R}$, we define $f\left(t^{-}\right):=\lim _{\tau \nearrow_{t}} f(\tau)$ and $f\left(t^{+}\right):=\lim _{\tau \searrow t} f(\tau)$, provided that these limits exist.

\section{B. Linear differential behaviors}

We call $\mathfrak{B} \subseteq \mathfrak{C}^{\infty}\left(\mathbb{R}, \mathbb{R}^{\mathrm{w}}\right)$ a linear time-invariant differential behavior if $\mathfrak{B}$ is the set of solutions of a finite system of constant-coefficient linear differential equations, i.e. if there exists a polynomial matrix $R \in \mathbb{R}^{\mathrm{g} \times \mathrm{w}}[\xi]$ such that $\mathfrak{B}=\left\{w \in \mathfrak{C}^{\infty}\left(\mathbb{R}, \mathbb{R}^{\mathrm{w}}\right) \mid R\left(\frac{d}{d t}\right) w=0\right\}=:$ ker $R\left(\frac{d}{d t}\right)$. If $\mathfrak{B}=\operatorname{ker} R\left(\frac{d}{d t}\right)$, then we call $R$ a kernel representation of $\mathfrak{B}$. We denote with $\mathfrak{L}^{\mathrm{w}}$ the set of all linear time-invariant differential behaviors with w variables.

Autonomous behaviors are defined as follows (see Ch. 3 of [4]).

Definition 1: $\mathfrak{B} \in \mathfrak{L}^{\mathrm{w}}$ is autonomous if for all $w_{1}, w_{2} \in$ $\mathfrak{B},\left\{w_{1}(t)=w_{2}(t)\right.$ for $\left.t<0\right\} \Longrightarrow\left\{w_{1}=w_{2}\right\}$.

It can be shown that if $\mathfrak{B}$ is autonomous, it admits a kernel representation with $R$ square and nonsingular. Moreover, it is finite-dimensional as a subspace of $\mathfrak{C}^{\infty}\left(\mathbb{R}, \mathbb{R}^{\mathrm{w}}\right)$, and its dimension equals $\operatorname{deg}(\operatorname{det}(R))$.

In this paper we use the notion of positive-realness [1].

Definition 2: A square matrix $B(\lambda)$ of rational functions is said to be positive-real if: all its entries are analytic in $\operatorname{Re}(\lambda)>0 ; B(\lambda)$ is real if $\lambda$ is real; and $B(-\lambda)^{\top}+B(\lambda) \geq 0$ for all $\operatorname{Re}(\lambda) \geq 0$.

The third condition of Definition 2 implies that

$$
B(-j \omega)^{\top}+B(j \omega) \geq 0 \forall \omega \in \mathbb{R} .
$$

If the inequality is strict, we call $B$ strictly positive-real. ${ }^{1}$

\section{Quadratic differential forms}

Let $\Phi \in \mathbb{R}^{\mathrm{w} \times \mathrm{w}}[\zeta, \eta]$ be a two-variable polynomial matrix. Without loss of generality we assume that $\Phi(\zeta, \eta)=$ $\Phi(\eta, \zeta)^{\top}$, i.e. that $\Phi(\zeta, \eta)$ is symmetric. We say that $\Phi(\zeta, \eta)$ has order $L$ if it can be written as $\Phi(\zeta, \eta)=$ $\sum_{k, \ell=0}^{L} \Phi_{k, \ell} \zeta^{k} \eta^{\ell}$, where $\Phi_{k, L}=\Phi_{L, k}$ is a nonzero matrix for some $k \in \mathbb{N}$. The quadratic differential form (QDF) $Q_{\Phi}$

\footnotetext{
${ }^{1}$ The definition of strictly positive real functions is not uniform in the literature; we refer to [10], Th. 2.1 .
} 
associated with $\Phi \in \mathbb{R}^{\mathrm{w} \times \mathrm{w}}[\zeta, \eta]$ is defined by

$$
\begin{aligned}
Q_{\Phi}: \mathfrak{C}^{\infty}\left(\mathbb{R}, \mathbb{R}^{\mathrm{w}}\right) & \longrightarrow \mathfrak{C}^{\infty}(\mathbb{R}, \mathbb{R}) \\
w & \mapsto Q_{\Phi}(w)=\sum_{k, \ell}\left(\frac{d^{k}}{d t^{k}} w\right)^{\top} \Phi_{k, \ell}\left(\frac{d^{\ell}}{d t^{\ell}} w\right) .
\end{aligned}
$$

We define the order of the quadratic differential form $Q_{\Phi}$ as the order of $\Phi(\zeta, \eta)$. Note that $\Phi(\zeta, \eta)=$ $S_{L}^{\mathrm{w}}(\zeta)^{\top} \widetilde{\Phi} S_{L}^{\mathrm{w}}(\eta)$, where $L$ is the order of $\Phi(\zeta, \eta), S_{L}^{\mathrm{w}}(\xi)^{\top}:=$

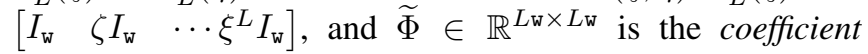
matrix of $\Phi$.

We say that a QDF $Q_{\Phi}$ is nonnegative along $\mathfrak{B}$, denoted $Q_{\Phi} \stackrel{\mathfrak{B}}{\geq} 0$, if $\left(Q_{\Phi}(w)\right)(t) \geq 0$ for all $w \in \mathfrak{B}$ and $t \in \mathbb{R}$. If a QDF $Q_{\Phi}$ is nonnegative for every trajectory in $\mathfrak{C}^{\infty}\left(\mathbb{R}, \mathbb{R}^{\mathrm{w}}\right)$ we write $Q_{\Phi} \geq 0$ and say that $Q_{\Phi}$ is nonnegative definite. Note that $\Phi$ is nonnegative definite if and only if $\widetilde{\Phi} \geq 0$. We say that $Q_{\Phi}$ is positive along $\mathfrak{B}$, denoted by $Q_{\Phi} \stackrel{\overline{\mathfrak{B}}}{>} 0$, if $Q_{\Phi} \stackrel{\mathfrak{B}}{\geq} 0$ and $Q_{\Phi}(w)=0, w \in \mathfrak{B}$, implies that $w=0$. A QDF is positive definite if it is positive along $\mathfrak{C}^{\infty}\left(\mathbb{R}, \mathbb{R}^{\mathrm{w}}\right)$; this happens if and only if $\widetilde{\Phi}>0$. We define $Q_{\Phi} \stackrel{\mathfrak{B}}{<} 0$, etc. in an analogous manner.

The derivative $\frac{d}{d t} Q_{\Phi}=: Q_{\dot{\Phi}}$ of a $\mathrm{QDF} Q_{\Phi}$ is also a QDF, and the associated two-variable polynomial matrix is $\dot{\Phi}(\zeta, \eta):=(\zeta+\eta) \Phi(\zeta, \eta)$ (see [12], section 3).

A Lyapunov function for a behavior $\mathfrak{B} \in \mathfrak{L}^{\mathrm{w}}$ is defined as a quadratic differential form $Q_{\Phi}$ whose values $Q_{\Phi}(w)$ are nonnegative and decrease with the time for all $w \in \mathfrak{B}$, i.e. $Q_{\Phi} \stackrel{\mathfrak{B}}{\geq} 0$ and $\frac{d}{d t} Q_{\Phi} \stackrel{\mathfrak{B}}{<} 0$.

The concept of $R$-canonical representative is employed for two-variable polynomial matrices. Let $R \in \mathbb{R}^{\mathrm{w} \times \mathrm{w}}[\xi]$ be nonsingular and $\Phi \in \mathbb{R}^{\mathrm{w} \times \mathrm{w}}[\zeta, \eta]$. Factorize $\Phi(\zeta, \eta)=$ $M(\zeta)^{\top} N(\eta)$ and compute the $R$-canonical representatives $M^{\prime}=M \bmod R$; and $N^{\prime}=N \bmod R$. Then the $R$-canonical representative of $\Phi(\zeta, \eta)$ is defined as $\Phi(\zeta, \eta) \bmod R:=M^{\prime}(\zeta)^{\top} N^{\prime}(\eta)$. In this sense, the QDFs $Q_{\Phi}, Q_{\Phi^{\prime}}$ are equivalent along ker $R\left(\frac{d}{d t}\right)$, which means that $Q_{\Phi^{\prime}}(w)=Q_{\Phi}(w)$ for all $w \in \operatorname{ker} R\left(\frac{d}{d t}\right)$.

\section{SWITCHED LINEAR DIFFERENTIAL SYSTEMS}

We recall the basic definitions of [6], [5].

Definition 3: A switched linear differential system (SLDS) $\Sigma$ is a quadruple $\Sigma=\{\mathcal{P}, \mathcal{F}, \mathcal{S}, \mathcal{G}\}$ where: $\mathcal{P}=\{1, \ldots, N\} \subset \mathbb{N}$ is the set of indices; $\mathcal{F}=\left(\mathfrak{B}_{1}, \ldots, \mathfrak{B}_{N}\right)$, with $\mathfrak{B}_{j} \in \mathfrak{L}^{\mathrm{w}}$ for $j \in \mathcal{P}$, is the bank of behaviors; $\mathcal{S}=\{s: \mathbb{R} \rightarrow \mathcal{P}\}$ with $s$ piecewise constant and right-continuous, is the set of admissible switching signals; and $\mathcal{G}=\left\{(k, \ell), G_{k \rightarrow \ell}^{+}(\xi), G_{k \rightarrow \ell}^{-}(\xi)\right\}$, where $\left(G_{k \rightarrow \ell}^{+}(\xi), G_{k \rightarrow \ell}^{-}(\xi)\right) \in\left(\mathbb{R}[\xi]^{\bullet \times \mathrm{w}}\right)^{2}$ and $(k, \ell) \in \mathcal{P} \times \mathcal{P}$, $k \neq \ell$, is the set of gluing conditions. For a given $s \in \mathcal{S}$, the set of switching instants with respect to $s$ is $\mathbb{T}_{s}:=\left\{t \in \mathbb{R} \mid \lim _{\tau} \nearrow_{t} s(\tau) \neq s(t)\right\}=\left\{t_{1}, t_{2}, \ldots\right\}$ where $t_{i}<t_{i+1}$.

We make the standard assumption that the switching signal is arbitrary and well-defined, i.e. every finite interval of $\mathbb{R}$ contains only a finite number of switching instants (see [9]).
Definition 4: Let $\Sigma$ be a SLDS and $s \in \mathcal{S}$. The $s$-switched behavior $\mathfrak{B}^{s}$ with respect to $\Sigma$ is the set of trajectories satisfying the following conditions: 1) for all $t_{i}, t_{i+1} \in \mathbb{T}_{s}$, there exists $k \in \mathcal{P}$ such that $w_{\left.\right|_{\left[t_{i}, t_{i+1}\right)}} \in \mathfrak{B}_{\left.k\right|_{\left[t_{i}, t_{i+1}\right)}}$;2) $w$ satisfies the gluing conditions $\mathcal{G}$ at the switching instants:

$$
\left(G_{s\left(t_{i-1}\right) \rightarrow s\left(t_{i}\right)}^{+}\left(\frac{d}{d t}\right)\right) w\left(t_{i}^{+}\right)=\left(G_{s\left(t_{i-1}\right) \rightarrow s\left(t_{i}\right)}^{-}\left(\frac{d}{d t}\right)\right) w\left(t_{i}^{-}\right),
$$

for each $t_{i} \in \mathbb{T}_{s}$.

The switched behavior $\mathfrak{B}^{\Sigma}$ of $\Sigma$ is defined by $\mathfrak{B}^{\Sigma}:=$ $\bigcup_{s \in \mathcal{S}} \mathfrak{B}^{s}$.

In the rest of this paper we consider scalar ( $w=1)$ behaviors, and "standard" gluing conditions which are defined as follows. Let $\Sigma$ be a SLDS and let $\mathfrak{B}_{k}:=\operatorname{ker} p_{k}\left(\frac{d}{d t}\right), \mathfrak{B}_{\ell}:=$ ker $p_{\ell}\left(\frac{d}{d t}\right)$ be a pair behaviors in $\mathcal{F}$, where $\left(p_{k}, p_{\ell}\right) \in \mathbb{R}[\xi]$ and $n_{k}:=\operatorname{deg}\left(p_{k}\right), n_{\ell}:=\operatorname{deg}\left(p_{\ell}\right)$. We define the standard gluing conditions when we switch from the behavior $\mathfrak{B}_{k}$ to $\mathfrak{B}_{\ell}$ for all $t_{i} \in \mathbb{T}_{s}$ as

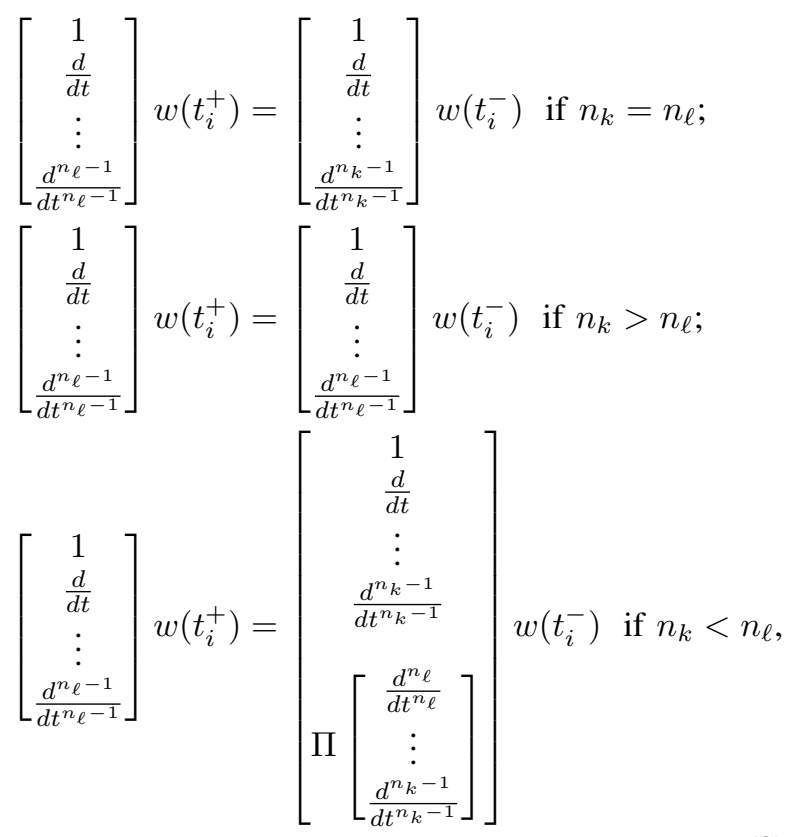

where $\Pi \in \mathbb{R}^{\left(n_{\ell}-n_{k}\right) \times n_{k}}$ is such that

$$
\left[\begin{array}{c}
\xi^{n_{k}} \\
\vdots \\
\xi^{n_{\ell}-1}
\end{array}\right] \bmod p_{k}=\Pi\left[\begin{array}{c}
1 \\
\vdots \\
\xi^{n_{k}-1}
\end{array}\right] \text {. }
$$

In words, when switching from a dynamical regime $\mathfrak{B}_{k}$ to $\mathfrak{B}_{\ell}$, we rewrite if necessary every derivative of $w$ of order higher than $n_{k}-1$ as a linear combination of derivatives of order at most $n_{k}-1$, according to the canonical representative of $\xi^{j}$ modulo $p_{k}, j=0, \ldots, n_{\ell}-1$, (see section II-A). Thus at every switching instant, the state of the active behavior is uniquely specified as a linear function of the state of the behavior before the switch, allowing the continuation of the trajectories of the switched behavior by providing a full set of "initial conditions" after the switch. We call a SLDS with such gluing conditions a standard switched linear differential system. 


\section{STABILITY AND POSITIVE-REALNESS}

Asymptotically stable SLDS are defined as follows.

Definition 5: A SLDS $\Sigma$ is asymptotically stable if $\lim _{t \rightarrow \infty} w(t)=0$ for all $w \in \mathfrak{B}^{\Sigma}$.

We prove the stability of a SLDS showing the existence of a Lyapunov function $Q_{\Psi}$, i.e. a QDF such that: $Q_{\Psi} \stackrel{\mathfrak{B}_{k}}{\geq} 0$ and $\frac{d}{d t} Q_{\Psi} \stackrel{\mathfrak{B}_{k}}{<} 0$ for all $k \in \mathcal{P}$; and the value of $Q_{\Psi}$ does not increase at the switching instants, i.e. $Q_{\Psi}(w)\left(t_{i}^{-}\right) \geq$ $Q_{\Psi}(w)\left(t_{i}^{+}\right)$for all $t_{i} \in \mathbb{T}_{s}$.

We summarize previous results (see [6], [5]) on the stability of SLDS with two behaviors in the following theorem.

Theorem 1: Let $p_{j} \in \mathbb{R}[\xi], j=1,2$, be Hurwitz polynomials, and define $n_{j}:=\operatorname{deg}\left(p_{j}\right), j=1,2$. Let $\mathcal{F}=$ $\left\{\mathfrak{B}_{1}, \mathfrak{B}_{2}\right\}$ with $\mathfrak{B}_{j}:=\operatorname{ker} p_{j}\left(\frac{d}{d t}\right), j=1,2$. Assume that $\frac{p_{2}}{p_{1}}$ is strictly positive-real with $n_{1} \geq n_{2}$. Define $x_{1}(\xi):=$ $\left[\begin{array}{lll}1 & \cdots & \xi^{n_{1}-1}\end{array}\right]^{\top}, x_{2}(\xi):=\left[\begin{array}{lll}1 & \cdots & \xi^{n_{2}-1}\end{array}\right]^{\top}$, and the set of gluing conditions $\mathcal{G}$ with $G_{2 \rightarrow 1}^{-}(\xi)=x_{1}(\xi) \bmod p_{2}$; $G_{2 \rightarrow 1}^{+}(\xi)=x_{1}(\xi)$; and $G_{1 \rightarrow 2}^{-}(\xi) \stackrel{=}{=} x_{2}(\xi)=G_{1 \rightarrow 2}^{+}(\xi)$. Define $\Phi(\zeta, \eta):=p_{1}(\zeta) p_{2}(\eta)+p_{2}(\zeta) p_{1}(\eta)$. Then, there exists a polynomial vector $d \in \mathbb{R}^{\bullet \times 1}[\xi]$ such that

1. $p_{1}(-\xi) p_{2}(\xi)+p_{2}(-\xi) p_{1}(\xi)=d(-\xi)^{\top} d(\xi)$.

2. $\Psi(\zeta, \eta):=\frac{\Phi(\zeta, \eta)-d(\zeta)^{\top} d(\eta)}{\zeta+\eta} \in \mathbb{R}[\zeta, \eta]$.

3. $Q_{\Psi}$ is a Lyapunov function for $\mathcal{F}$.

Proof: See [6] Theorem 10, and [5] Theorem 2.3.

As shown in [13] Th. 5.10, if we assume that $\frac{p_{2}}{p_{1}}$ is strictly positive-real, then the degree of $p_{1}$ and $p_{2}$ cannot differ by more than one, consequently, Theorem 1 only covers the situation where $n_{1}-n_{2}=0$ or $n_{1}-n_{2}=1$. To study the stability of behaviors whose state space dimension differs arbitrarily, we introduce the concept of positive-real completion.

Definition 6: Let $\Sigma$ be a standard SLDS. The polynomial $m \in \mathbb{R}[\xi]$ is a strictly positive-real completion of $\frac{p_{2}}{p_{1}}$ if $\frac{m p_{2}}{p_{1}}$ is strictly proper and strictly positive-real.

Remark 1: Not every pair of Hurwitz polynomials has a strictly- positive-real completion, for example the polynomials $p_{1}(\xi):=2523677+435616 \xi+81559 \xi^{2}+7000 \xi^{3}+$ $603 \xi^{4}+24 \xi^{5}+\xi^{6}$ and $p_{2}(\xi):=65+46 \xi+26 \xi^{2}+6 \xi^{3}+\xi^{4}$.

Remark 2: Strictly- positive-real completions are not unique; for instance the rational function $\frac{m p_{2}}{p_{1}}$ with $p_{1}(\xi):=$ $(\xi+1)(\xi+3)(\xi+6)$ and $p_{2}:=\xi+2$ is positive-real with $m$ equal to $\xi+4, \xi+5$ and many other options.

\section{A. Computation of a positive-real completion}

To compute a strictly-proper positive-real completion $m$ we can use the positive-real lemma [1]. Define $p_{3}:=$ $m p_{2}$ and $n_{3}:=\operatorname{deg}\left(p_{3}\right)$; in the following we assume that $n_{1}=n_{3}+1$. A realization $(A, B, C, 0)$ of $\frac{p_{3}(\xi)}{p_{1}(\xi)}$ can be written in controllable canonical form, i.e. $A x(\xi):=$ $\xi x(\xi) \bmod p_{1}=\xi x(\xi)-B p_{1}(\xi)$, and $p_{3}(\xi)=C x(\xi)$, where $x(\xi)=\left[\begin{array}{lll}1 & \cdots & \xi^{n_{1}-1}\end{array}\right]^{\top}$. The coefficients of $m$ are parameters to be determined, so we write

$$
C^{\top}:=\underbrace{\left[\begin{array}{ccccc}
p_{2,0} & 0 & 0 & \cdots & 0 \\
p_{2,1} & p_{2,0} & 0 & \cdots & 0 \\
\vdots & \vdots & \ddots & \cdots & \vdots
\end{array}\right]}_{=: \tilde{T}} \underbrace{\left[\begin{array}{c}
m_{0} \\
m_{1} \\
\vdots \\
m_{n_{1}-n_{2}-1}
\end{array}\right]}_{=: \tilde{m}}
$$

where $\tilde{T} \in \mathbb{R}^{n_{1} \times\left(n_{1}-n_{2}\right)}$ is a Töplitz matrix containing the coefficients $p_{2, j}$ of $p_{2}(\xi)$; and $\tilde{m} \in R^{\left(n_{1}-n_{2}\right) \times 1}$ contains the unknown coefficients of $m(\xi)$.

Now if for some $\varepsilon \geq 0$ and for some $m_{i}, i=0, \ldots, n_{1}-$ $n_{2}-1$, the inequality

$$
\left[\begin{array}{cc}
A^{\top} \tilde{\Psi}+\tilde{\Psi} A+2 \varepsilon \tilde{\Psi} & \tilde{\Psi} B-C^{\top} \\
B^{\top} \tilde{\Psi}-C & 0
\end{array}\right] \leq 0,
$$

has a positive-definite solution $\tilde{\Psi}=\tilde{\Psi}^{\top} \in \mathbb{R}^{n_{1} \times n_{1}}$, then $G(\xi)=\frac{p_{3}(\xi)}{p_{1}(\xi)}=C(\xi I-A)^{-1} B$ is strictly positive-real, and $m$ is a completion. The LMI (4) can be solved using standard computational methods. On the other hand, if (4) has no solution, we conclude that the pair $p_{1}, p_{2}$ does not have a positive-real completion, see Remark 1.

\section{B. Stability of SLDS using positive-real completions}

In the following section we analyse some further consequences of the existence of positive-real completions.

\section{MAIN RESULTS}

To discuss the main results of this paper we need to illustrate first an important structural property of a Lyapunov function $Q_{\Psi}$ for a $\operatorname{SLDS} \Sigma$ with $\mathcal{F}:=\left\{\mathfrak{B}_{i}:=\right.$ ker $\left.p_{i}\left(\frac{d}{d t}\right)\right\}_{i=1,2}$ with $p_{i} \in \mathbb{R}[\xi], i=1,2$, and gluing conditions as in (2). Let $\Psi(\zeta, \eta)$ induce a Lyapunov function for a standard SLDS as in def. 4, and write

$$
\Psi(\zeta, \eta)=\left[\begin{array}{lll}
1 & \cdots & \zeta^{n_{1}-1}
\end{array}\right] \underbrace{\left[\begin{array}{ll}
\Psi_{11} & \Psi_{12} \\
\Psi_{12}^{\top} & \Psi_{22}
\end{array}\right]}_{=: \widetilde{\Psi}}\left[\begin{array}{c}
1 \\
\vdots \\
\eta^{n_{1}-1}
\end{array}\right],
$$

for suitable matrices $\Psi_{11} \in \mathbb{R}^{n_{2} \times n_{2}}, \Psi_{12} \in \mathbb{R}^{n_{2} \times\left(n_{1}-n_{2}\right)}$ and $\Psi_{22} \in \mathbb{R}^{\left(n_{1}-n_{2}\right) \times\left(n_{1}-n_{2}\right)}$. Note that since $Q_{\Psi}$ is positive along $\mathfrak{B}_{1}$, the coefficient matrix

$$
\tilde{\Psi}:=\left[\begin{array}{ll}
\Psi_{11} & \Psi_{12} \\
\Psi_{12}^{\top} & \Psi_{22}
\end{array}\right]
$$

is positive definite. Now consider the following Lemma.

Lemma 1: Let $\Sigma$ be SLDS with $\mathcal{F}:=\left\{\mathfrak{B}_{i}:=\right.$ ker $\left.p_{i}\left(\frac{d}{d t}\right)\right\}_{i=1,2}$ with $p_{i} \in \mathbb{R}[\xi], i=1,2$, and gluing conditions as in (2). Define $n_{i}:=\operatorname{deg}\left(p_{i}\right), i=1,2$ and assume that $n_{1}>n_{2}$. Assume that there exists a Lyapunov function $Q_{\Psi}$ for $\Sigma$ and let its coefficient matrix $\tilde{\Psi}$ be partitioned as in (5), then $\Psi_{12}=-\Pi^{\top} \Psi_{22}$.

Proof: In order to prove the claim, define $z:=$ $\left[\begin{array}{lll}w & \cdots & \frac{d^{n_{2}-1}}{d t^{n_{2}-1}} w\end{array}\right]^{\top}$ and $v:=\left[\begin{array}{lll}\frac{d^{n_{2}}}{d t^{n_{2}}} w & \cdots & \frac{d^{n_{1}-1}}{d t^{n_{1}-1}} w\end{array}\right]^{\top}$, 
then taking the standard gluing conditions (2) into account, when switching from $\mathfrak{B}_{1}$ to $\mathfrak{B}_{2}$ at $t_{k}$, the inequality $Q_{\Psi}(w)\left(t_{k}^{-}\right)-Q_{\Psi}(w)\left(t_{k}^{+}\right) \geq 0$ holds true if and only if

$$
\left[\begin{array}{l}
z\left(t_{k}^{-}\right) \\
v\left(t_{k}^{-}\right)
\end{array}\right]^{\top}\left(\tilde{\Psi}-\left[\begin{array}{cc}
I_{n_{2}} & \Pi^{\top} \\
0 & 0
\end{array}\right] \tilde{\Psi}\left[\begin{array}{cc}
I_{n_{2}} & 0 \\
\Pi & 0
\end{array}\right]\right)\left[\begin{array}{l}
z\left(t_{k}^{-}\right) \\
v\left(t_{k}^{-}\right)
\end{array}\right] \geq 0 .
$$

Since $\left[z^{\top}\left(t_{k}^{-}\right) \quad v^{\top}\left(t_{k}^{-}\right)\right]$is arbitrary in $\mathbb{R}^{n_{1}}$ for the trajectories of $\Sigma$, the last equality implies that

$$
\tilde{\Psi}-\left[\begin{array}{cc}
I_{n_{2}} & \Pi^{\top} \\
0 & 0
\end{array}\right] \tilde{\Psi}\left[\begin{array}{cc}
I_{n_{2}} & 0 \\
\Pi & 0
\end{array}\right] \geq 0
$$

After standard linear algebra manipulations we find that (6) is equivalent to

$$
\left[\begin{array}{cc}
-\left(\Psi_{12}+\Pi^{\top} \Psi_{22}\right) \Psi_{22}^{-1}\left(\Psi_{12}^{\top}+\Psi_{22} \Pi\right) & 0 \\
0 & \Psi_{22}
\end{array}\right] \geq 0 .
$$

Now consider that the $(1,1)$ block in $(7)$ is negative semidefinite; consequently, (7) holds if and only if the $(1,1)$ block is zero, i.e. if and only if $\Psi_{12}=-\Pi^{\top} \Psi_{22}$. The claim is proved.

\section{A. Positive-realness and stability of SLDS with three behav-} iors

We now prove a sufficient condition for the asymptotic stability of a SLDS with three behaviors.

Theorem 2: Let $p_{i} \in \mathbb{R}[\xi], i=1,2$, be Hurwitz polynomials such that $\operatorname{deg}\left(p_{1}\right)>\operatorname{deg}\left(p_{2}\right)$. Assume that there exists $m \in \mathbb{R}[\xi]$, with $\operatorname{deg}(m)=\operatorname{deg}\left(p_{1}\right)+1$, and a Lyapunov function $Q_{\Psi}$ for ker $p_{i}\left(\frac{d}{d t}\right), i=1,2$, as in Lemma 1 , such that the coefficient matrices $\tilde{m}$ and $\tilde{\Psi}$ satisfy the LMI (4) with $C$ as in (3). Define $p_{3}(\xi):=m(\xi) p_{2}(\xi)$, $\mathfrak{B}_{j}:=\operatorname{ker} p_{j}\left(\frac{d}{d t}\right), j=1,2,3$, and denote $n_{j}:=\operatorname{deg}\left(p_{j}\right)$, $j=1,2,3$. Moreover, define $x_{2}(\xi):=\left[\begin{array}{lll}1 & \cdots & \xi^{n_{2}-1}\end{array}\right]^{\top}$; $x_{3}^{\prime}(\xi):=\left[\begin{array}{lll}\xi^{n_{2}} & \cdots & \xi^{n_{3}-1}\end{array}\right]^{\top}, x_{3}:=\left[\begin{array}{ll}x_{2}(\xi) & x_{3}^{\prime}(\xi)\end{array}\right]^{\top}$ and $x_{1}^{\prime}(\xi):=\xi^{n_{1}-1}$.

Consider the $\operatorname{SLDS} \Sigma^{\prime}$ with $\mathcal{F}^{\prime}=\left(\mathfrak{B}_{1}, \mathfrak{B}_{2}, \mathfrak{B}_{3}\right)$ and gluing conditions

$$
\begin{gathered}
\left(G_{2 \rightarrow 1}^{-}(\xi), G_{2 \rightarrow 1}^{+}(\xi)\right):=\left(\left[\begin{array}{c}
x_{2}(\xi) \\
\Pi_{1} x_{2}(\xi)
\end{array}\right],\left[\begin{array}{l}
x_{2}(\xi) \\
x_{3}^{\prime}(\xi) \\
x_{1}^{\prime}(\xi)
\end{array}\right]\right) \\
\left(G_{1 \rightarrow 2}^{-}(\xi), G_{1 \rightarrow 2}^{+}(\xi)\right):=\left(x_{2}(\xi), x_{2}(\xi)\right) \\
\left(G_{3 \rightarrow 1}^{-}(\xi), G_{3 \rightarrow 1}^{+}(\xi)\right):=\left(\left[\begin{array}{c}
x_{3}(\xi) \\
\Pi_{3} x_{3}(\xi)
\end{array}\right],\left[\begin{array}{l}
x_{3}(\xi) \\
x_{1}^{\prime}(\xi)
\end{array}\right]\right) \\
\left(G_{1 \rightarrow 3}^{-}(\xi), G_{1 \rightarrow 3}^{+}(\xi)\right):=\left(x_{3}(\xi), x_{3}(\xi)\right) \\
\left(G_{2 \rightarrow 3}^{-}(\xi), G_{2 \rightarrow 3}^{+}(\xi)\right):=\left(\left[\begin{array}{c}
x_{2}(\xi) \\
\Pi_{2} x_{2}(\xi)
\end{array}\right],\left[\begin{array}{l}
x_{2}(\xi) \\
x_{3}^{\prime}(\xi)
\end{array}\right]\right) \\
\left(G_{3 \rightarrow 2}^{-}(\xi), G_{3 \rightarrow 2}^{+}(\xi)\right):=\left(x_{2}(\xi), x_{2}(\xi)\right)
\end{gathered}
$$

where $\Pi_{1} \in \mathbb{R}^{\left(n_{1}-n_{2}\right) \times n_{2}}, \Pi_{2} \in \mathbb{R}^{\left(n_{3}-n_{2}\right) \times n_{2}}, \Pi_{3} \in$ $\mathbb{R}^{\left(n_{1}-n_{3}\right) \times n_{3}}$ are such that $\left[\begin{array}{l}x_{3}^{\prime}(\xi) \\ x_{1}^{\prime}(\xi)\end{array}\right] \bmod p_{2}=\Pi_{1} x_{2}(\xi) ;$ $x_{3}^{\prime}(\xi) \bmod p_{2}=\Pi_{2} x_{2}(\xi)$; and $x_{1}^{\prime}(\xi) \bmod p_{3}=\Pi_{3} x_{3}(\xi)$. Then there exists a Lyapunov function $Q_{\Psi}$ for $\mathcal{F}^{\prime}$.
Proof: In order to show that $Q_{\Psi}$ is a Lyapunov function for $\mathcal{F}^{\prime}$, we prove the following statements:

S1. $Q_{\Psi} \stackrel{\mathfrak{B}_{1}}{\geq} 0$ and $\frac{d}{d t} Q_{\Psi} \stackrel{\mathfrak{B}_{1}}{<} 0$.

S2. $Q_{\Psi} \stackrel{\mathfrak{B}_{2}}{\geq} 0$ and $\frac{d}{d t} Q_{\Psi} \stackrel{\mathfrak{B}_{2}}{<} 0$.

S3. $Q_{\Psi} \stackrel{\mathfrak{B}_{3}}{\geq} 0$ and $\frac{d}{d t} Q_{\Psi} \stackrel{\mathfrak{B}_{3}}{<} 0$;

and moreover, we show that the value of $Q_{\Psi}$ does not increase at the switching instants, i.e.

S4. when we switch from $\mathfrak{B}_{1}$ to $\mathfrak{B}_{2}$ and viceversa.

S5. when we switch from $\mathfrak{B}_{1}$ to $\mathfrak{B}_{3}$ and viceversa.

S6. when we switch from $\mathfrak{B}_{3}$ to $\mathfrak{B}_{2}$ and viceversa.

Note that statements $\mathbf{S 1}$ and $\mathbf{S 2}$ and $\mathbf{S 4}$ hold, since $Q_{\Psi}$ is a Lyapunov function for $\left\{\mathfrak{B}_{1}, \mathfrak{B}_{2}\right\}$.

In order to prove $\mathbf{S 3}$, define $\Psi_{3}(\zeta, \eta):=\Psi(\zeta, \eta) \bmod p_{3}$. Note that since $Q_{\Psi} \geq 0$ and $Q_{\Psi} \stackrel{\mathfrak{B}_{3}}{=} Q_{\Psi_{3}}$, it follows that $Q_{\Psi_{3}} \geq 0$. To prove the second part of the statement, since $\frac{p_{3}}{p_{1}}$ is strictly positive-real, then

$$
(\zeta+\eta) \Psi(\zeta, \eta)=p_{1}(\zeta) p_{3}(\eta)+p_{3}(\zeta) p_{1}(\eta)-d(\zeta)^{\top} d(\eta)
$$

for some polynomial vector $d \in \mathbb{R}^{\bullet \times 1}[\xi]$ (see Theorem 1, section IV). From standard results in the theory of quadratic differential forms (see [12], p.1716), we know that the derivative of $Q_{\Psi_{3}}$ is induced by the two variable polynomial $(\zeta+\eta) \Psi(\zeta, \eta) \bmod p_{3}=-d^{\prime}(\zeta)^{\top} d^{\prime}(\eta)$, where $d^{\prime}:=d \bmod p_{3}$. Therefore, to prove that the derivative of $Q_{\Psi_{3}}$ decreases along $\mathfrak{B}_{3}$ it is enough to check that $\operatorname{col}\left(d^{\prime}(\lambda), p_{3}(\lambda)\right)$ is full column rank for all $\lambda \in \mathbb{C}$, which guarantees that $\frac{d}{d t}\left(Q_{\Psi_{3}}(w)\right)$ is non zero for the trajectories of $\mathfrak{B}_{3}$. By contradiction, assume that there exists $\lambda \in \mathbb{C}$ such that $p_{1}(\lambda)=0$ and $d(\lambda)=0$. Note that since $p_{1}$ is Hurwitz necessarily $\lambda \in \mathbb{C}_{-}$, the open left half-plane. Substitute $\zeta=\bar{\lambda}$ and $\eta=\lambda$ in the expression in (8), obtaining $(\bar{\lambda}+\lambda) \Psi(\bar{\lambda}, \lambda)=0$. Since $\lambda \in \mathbb{C}_{-}$, this is equivalent with $\Psi(\bar{\lambda}, \lambda)=0$, which implies that $\widetilde{\Psi}$ is not positive-definite, a contradiction.

The validity of statement $\mathbf{S 5}$ follows from Th. 1, since $\frac{p_{3}}{p_{1}}$ is strictly positive-real and $\operatorname{deg}\left(p_{3}\right)=\operatorname{deg}\left(p_{1}\right)-1$.

It remains to prove S6. When we switch from $\mathfrak{B}_{3}$ to $\mathfrak{B}_{2}$, the condition $Q_{\Psi}(w)\left(t_{i}^{-}\right)-Q_{\Psi}(w)\left(t_{i}^{+}\right) \geq 0$ must be satisfied. Since

$$
\left(\left[\begin{array}{l}
x_{2}(\xi) \\
x_{3}^{\prime}(\xi) \\
x_{1}^{\prime}(\xi)
\end{array}\right] \bmod p_{3}\right) \bmod p_{2}=\left[\begin{array}{l}
x_{2}(\xi) \\
x_{3}^{\prime}(\xi) \\
x_{1}^{\prime}(\xi)
\end{array}\right] \bmod p_{2}
$$

the condtion can be written as

$$
Q_{\Psi \bmod p_{3}}(w)-Q_{\left(\Psi \bmod p_{3}\right) \bmod p_{2}}(w) \geq 0 .
$$

In the following, we aim to express condition (9) in terms of a matrix inequality. We proceed by expressing the relation between $\Pi_{1}, \Pi_{2}$ and $\Pi_{3}$, and we first compute

$$
\left[\begin{array}{l}
x_{2}(\xi) \\
x_{3}^{\prime}(\xi) \\
x_{1}^{\prime}(\xi)
\end{array}\right] \bmod p_{2}=\left[\begin{array}{c}
x_{2}(\xi) \\
\Pi_{1} x_{2}(\xi)
\end{array}\right]
$$


Partition $\Pi_{3}:=\left[\begin{array}{ll}\Pi_{3}^{\prime} & \Pi_{3}^{\prime \prime}\end{array}\right]$ with $\Pi_{3}^{\prime} \in \mathbb{R}^{\left(n_{1}-n_{3}\right) \times n_{2}}$ and $\Pi_{3}^{\prime \prime} \in \mathbb{R}^{\left(n_{1}-n_{3}\right) \times\left(n_{3}-n_{2}\right)}$, then

$$
\left[\begin{array}{c}
x_{2}(\xi) \\
x_{3}^{\prime}(\xi) \\
x_{1}^{\prime}(\xi)
\end{array}\right] \bmod p_{3}=\left[\begin{array}{c}
x_{2}(\xi) \\
\Pi_{2} x_{2}(\xi) \\
\Pi_{3}^{\prime} x_{2}(\xi)+\Pi_{3}^{\prime \prime} x_{3}^{\prime}(\xi)
\end{array}\right]
$$

Consequently

$$
\left.\left(\left[\begin{array}{l}
x_{2}(\xi) \\
x_{3}^{\prime}(\xi) \\
x_{1}^{\prime}(\xi)
\end{array}\right] \bmod p_{3}\right) \bmod p_{2}=\left[\begin{array}{c}
x_{2}(\xi) \\
\Pi_{2} \\
\Pi_{3}^{\prime}+\Pi_{3}^{\prime \prime} \Pi_{2}
\end{array}\right] x_{2}(\xi)\right] .
$$

By comparing equations (10) and (11) we have that $\Pi_{1}=$ $\left[\begin{array}{c}\Pi_{2} \\ \Pi_{3}^{\prime}+\Pi_{3}^{\prime \prime} \Pi_{2}\end{array}\right]$. Now consider the coefficient matrix of the Lyapunov function $Q_{\Psi}$ and partition it as

$$
\tilde{\Psi}:=\left[\begin{array}{lll}
\Psi_{11} & \Psi_{12} & \Psi_{13} \\
\Psi_{12}^{\top} & \Psi_{22} & \Psi_{23} \\
\Psi_{13}^{\top} & \Psi_{23}^{\top} & \Psi_{33}
\end{array}\right]
$$

with $\Psi_{11} \in \mathbb{R}^{n_{2} \times n_{2}}, \quad \Psi_{12} \in \mathbb{R}^{n_{2} \times\left(n_{3}-n_{2}\right)}$, $\Psi_{13} \in \mathbb{R}^{n_{2} \times\left(n_{1}-n_{3}\right)}, \quad \Psi_{22} \in \mathbb{R}^{\left(n_{3}-n_{2}\right) \times\left(n_{3}-n_{2}\right)}$, $\Psi_{23} \in \mathbb{R}^{\left(n_{3}-n_{2}\right) \times\left(n_{1}-n_{3}\right)}$ and $\Psi_{33} \in \mathbb{R}^{\left(n_{1}-n_{3}\right) \times\left(n_{1}-n_{3}\right)}$. From the results of Lemma 1, since the Lyapunov function $Q_{\Psi}$ does not increase when switching from $\mathfrak{B}_{1}$ to $\mathfrak{B}_{2}$, this implies that

$$
\begin{aligned}
{\left[\begin{array}{l}
\Psi_{12}^{\top} \\
\Psi_{13}^{\top}
\end{array}\right] } & =-\left[\begin{array}{ll}
\Psi_{22} & \Psi_{23} \\
\Psi_{23}^{\top} & \Psi_{33}
\end{array}\right] \Pi_{1} \\
& =-\left[\begin{array}{ll}
\Psi_{22} & \Psi_{23} \\
\Psi_{23}^{\top} & \Psi_{33}
\end{array}\right]\left[\begin{array}{c}
\Pi_{2} \\
\Pi_{3}^{\prime}+\Pi_{3}^{\prime \prime} \Pi_{2}
\end{array}\right]
\end{aligned}
$$

and consequently

$$
\Psi_{12}^{\top}=-\left(\Psi_{22} \Pi_{2}+\Psi_{23} \Pi_{3}^{\prime}+\Psi_{23} \Pi_{3}^{\prime \prime} \Pi_{2}\right),
$$

and

$$
\Psi_{13}^{\top}=-\left(\Psi_{23} \Pi_{2}+\Psi_{33} \Pi_{3}^{\prime}+\Psi_{33} \Pi_{3}^{\prime \prime} \Pi_{2}\right) .
$$

The following lemma provides important structural properties of $Q_{\Psi \bmod p_{3}}$ that will be essential for the rest of the proof.

Lemma 2: Let $Q_{\Psi}$, its coefficient matrix $\tilde{\Psi}$ and $\Pi_{3}:=$ $\left[\begin{array}{ll}\Pi_{3}^{\prime} & \Pi_{3}^{\prime \prime}\end{array}\right]$, be as previously defined and let $\tilde{\tilde{\Psi}}$ be the coefficient matrix of $Q_{\Psi \bmod p_{3}}$. Consider the partition

$$
\tilde{\tilde{\Psi}}:=\left[\begin{array}{ll}
\tilde{\tilde{\Psi}}_{11} & \tilde{\tilde{\Psi}}_{12} \\
\tilde{\tilde{\Psi}}_{12}^{\top} & \tilde{\tilde{\Psi}}_{22}
\end{array}\right]
$$

with $\tilde{\tilde{\Psi}}_{11} \in \mathbb{R}^{n_{2} \times n_{2}}, \tilde{\tilde{\Psi}}_{12} \in \mathbb{R}^{n_{2} \times\left(n_{3}-n_{2}\right)}$ and $\tilde{\tilde{\Psi}}_{22} \in$ $\mathbb{R}^{\left(n_{3}-n_{2}\right) \times\left(n_{3}-n_{2}\right)}$. Then

$$
\begin{aligned}
& \tilde{\tilde{\Psi}}_{11}=\left(\Psi_{11}+\Pi_{3}^{\prime} \Psi_{13}^{\top}+\Psi_{13} \Pi_{3}^{\prime}+\Pi_{3}^{\prime \top} \Psi_{33} \Pi_{3}^{\prime}\right), \\
& \tilde{\tilde{\Psi}}_{12}=\left(\Psi_{12}+\Pi_{3}^{\prime \top} \Psi_{23}^{\top}+\Psi_{13} \Pi_{3}^{\prime \prime}+\Pi_{3}^{\prime \top} \Psi_{33} \Pi_{3}^{\prime \prime}\right), \\
& \tilde{\tilde{\Psi}}_{22}=\left(\Psi_{22}+\Pi_{3}^{\prime \prime \top} \Psi_{23}^{\top}+\Psi_{23} \Pi_{3}^{\prime}+\Pi_{3}^{\prime \prime \top} \Psi_{33} \Pi_{3}^{\prime \prime}\right) .
\end{aligned}
$$

Proof: Following the same procedure as in Lemma 1 and considering the partitions (12) and (15), we conclude that the coefficient matrix of $Q_{\Psi \bmod p_{3}}$ is

$$
\begin{aligned}
& {\left[\begin{array}{ll}
\tilde{\tilde{\Psi}}_{11} & \tilde{\tilde{\Psi}}_{12} \\
\tilde{\tilde{\Psi}}_{12}^{\top} & \tilde{\tilde{\Psi}}_{22}
\end{array}\right]=} \\
& {\left[\begin{array}{cc}
I_{n_{2}} & 0 \\
0 & I_{\left(n_{3}-n_{2}\right)} \\
\Pi_{3}^{\prime} & \Pi_{3}^{\prime \prime}
\end{array}\right]^{\top}\left[\begin{array}{lll}
\Psi_{11} & \Psi_{12} & \Psi_{13} \\
\Psi_{12}^{\top} & \Psi_{22} & \Psi_{23} \\
\Psi_{13}^{\top} & \Psi_{23}^{\top} & \Psi_{33}
\end{array}\right]\left[\begin{array}{cc}
I_{n_{2}} & 0 \\
0 & I_{\left(n_{3}-n_{2}\right)} \\
\Pi_{3}^{\prime} & \Pi_{3}^{\prime \prime}
\end{array}\right] .}
\end{aligned}
$$

The desired equalities follow by inspection.

Now we return to the proof of the main Theorem. Note that from the inequality (9) we can obtain

$$
\left[\begin{array}{cc}
\tilde{\tilde{\Psi}}_{11} & \tilde{\tilde{\Psi}}_{12} \\
\tilde{\tilde{\Psi}}_{12}^{\top} & \tilde{\tilde{\Psi}}_{22}
\end{array}\right]-\left[\begin{array}{cc}
I_{n_{2}} & \Pi_{2}^{\top} \\
0 & 0
\end{array}\right]\left[\begin{array}{cc}
\tilde{\tilde{\Psi}}_{11} & \tilde{\tilde{\Psi}}_{12} \\
\tilde{\tilde{\Psi}}_{12}^{\top} & \tilde{\tilde{\Psi}}_{22}
\end{array}\right]\left[\begin{array}{cc}
I_{n_{2}} & 0 \\
\Pi_{2} & 0
\end{array}\right] \geq 0 .
$$

Note that similarly to Lemma 1 , this inequality holds if and only if $\tilde{\tilde{\Psi}}_{12}^{\top}+\tilde{\widetilde{\Psi}}_{22} \Pi_{2}=0$, or equivalently from Lemma 2 , the condition is satisfied if and only if

$$
\begin{aligned}
& \Psi_{12}^{\top}+\Pi_{3}^{\prime \prime \top} \Psi_{13}^{\top}+\Psi_{23} \Pi_{3}^{\prime}+\Pi_{3}^{\prime \prime \top} \Psi_{33} \Pi_{3}^{\prime}= \\
& -\left(\Psi_{22}+\Pi_{3}^{\prime \prime \top} \Psi_{23}^{\top}+\Psi_{23} \Pi_{3}^{\prime}+\Pi_{3}^{\prime \prime \top} \Psi_{33} \Pi_{3}^{\prime \prime}\right) \Pi_{2} .
\end{aligned}
$$

Substituting (14) in the latter equation we obtain (13) and we conclude that

$$
\left\{\left[\begin{array}{l}
\Psi_{12}^{\top} \\
\Psi_{13}^{\top}
\end{array}\right]=-\left[\begin{array}{ll}
\Psi_{22} & \Psi_{23} \\
\Psi_{23}^{\top} & \Psi_{33}
\end{array}\right] \Pi_{1}\right\} \Longrightarrow\left\{\tilde{\tilde{\Psi}}_{12}^{\top}=-\tilde{\tilde{\Psi}}_{22} \Pi_{2}\right\} .
$$

Consequently $Q_{\Psi}$ does not increase when switching from $\mathfrak{B}_{3}$ to $\mathfrak{B}_{2}$. It is a matter of straighforward verification to check that when we switch from $\mathfrak{B}_{2}$ to $\mathfrak{B}_{3}$ the value of $Q_{\Psi}$ remains the same before and after the switch. This concludes the proof of the Theorem.

Theorem 2 shows that the existence of a strictly positivereal completion $m$ associated to a SLDS $\Sigma$ with two behaviors $\mathfrak{B}_{j}:=\operatorname{ker} p_{j}\left(\frac{d}{d t}\right), j=1,2$, in the bank $\mathcal{F}$, implies the existence of a third behavior $\mathfrak{B}_{3}:=\operatorname{ker} p_{3}\left(\frac{d}{d t}\right)$ with $p_{3}:=m p_{2}$, in an augmented bank $\mathcal{F}^{\prime}$ of a $\operatorname{SLDS} \Sigma^{\prime}$. We defined the standard gluing conditions for $\Sigma^{\prime}$, associated to the switching among the behaviors $\mathfrak{B}_{i}, i=1,2,3$, as in (2) following that $n_{1}>n_{3}>n_{2}$. Consequently, the stability conditions derived from the analysis of the switching between the behaviors in $\mathcal{F}$ are compatible with the stability conditions for $\mathcal{F}^{\prime}$ concluding that if $\Sigma$ is asymptotically stable, so is $\Sigma^{\prime}$.

\section{B. Positive-realness and stability of families of three- behaviors}

Another consequence of the notion of positive-real completion is given in the following Theorem.

Theorem 3: Let $\Sigma^{\prime}$ be a SLDS as in Theorem 2. Assume that there exist two different strictly positive-real completions $m_{1}$ and $m_{2}$ for $\frac{p_{2}}{p_{1}}$, and let $\alpha \in[0,1]$. Then $m_{\alpha}:=\alpha m_{1}+$ $(1-\alpha) m_{2}$ is also a completion, i.e. $\frac{m_{\alpha} p_{2}}{p_{1}}$ is strictly positivereal. 
Moreover, define

$$
\mathfrak{F}_{\alpha}^{\prime}:=\left\{\operatorname{ker} p_{1}\left(\frac{d}{d t}\right), \operatorname{ker} p_{2}\left(\frac{d}{d t}\right), \operatorname{ker} p_{3, \alpha}\left(\frac{d}{d t}\right)\right\},
$$

with $p_{3, \alpha}:=m_{\alpha} p_{2}$ and the standard gluing conditions as in Theorem 2. Then $\mathfrak{F}_{\alpha}$ is stable.

Proof: The fact that $m_{\alpha}$ for all $\alpha \in[0,1]$ is strictly positive-real follows from straightforward computations:

$$
\begin{aligned}
& \frac{m_{\alpha}(-j \omega) p_{2}(-j \omega)}{p_{1}(-j \omega)}+\frac{m_{\alpha}(j \omega) p_{2}(j \omega)}{p_{1}(j \omega)} \\
= & \frac{\left(\alpha m_{1}(-j \omega)+(1-\alpha) m_{2}(-j \omega)\right) p_{2}(-j \omega)}{p_{1}(-j \omega)} \\
& +\frac{\left(\alpha m_{1}+(1-\alpha) m_{2}\right) p_{2}(j \omega)}{p_{1}(j \omega)} \\
= & \alpha \underbrace{\left(\frac{m_{1}(-j \omega) r_{2}(-j \omega)}{p_{1}(-j \omega)}+\frac{m_{1} p_{2}(j \omega)}{p_{1}(j \omega)}\right)}_{>0 \text { for all } \omega \in \mathbb{R}} \\
& +(1-\alpha) \underbrace{\left(\frac{m_{2}(-j \omega) p_{2}(-j \omega)}{p_{1}(j \omega)}\right)}_{p_{1}(-j \omega)},
\end{aligned}
$$

To prove that $\mathfrak{F}_{\alpha}$ is stable, use Theorem 2 .

Theorem 3 shows that the existence of two separate completions allows to establish the stability of a whole family of parameter-dependent SLDS with three behaviors $\mathfrak{F}_{\alpha}$. This result also shows that the asymptotic stability of a completion established in Theorem 2 is robust: perturbations of a given completion, parametrized by $\alpha$ as in Theorem 3, also result in a stable SLDS.

We now provide a method to compute more than one strictly- positive-real completion; the intuition behind this procedure is to consider small perturbations of a positivereal completion that result in other completions satisfying the frequency domain inequality (1).

Consider the realization $(A, B, C, 0)$ associated to a strictly positive real function $G(\xi):=C(\xi I-A)^{-1} B$ in section IV-A, and the LMI (4) with $C$ as in (3). Consider that $G(\xi-\varepsilon)$ is strictly positive-real for some constant $\varepsilon>0$ (see [10], Th. 3.3). We can use this fact to numerically compute different solutions $\tilde{m}$ and $\tilde{\Psi}$ for a given pair of polynomials $\left(p_{1}, p_{2}\right)$ by defining different values of $\varepsilon \geq 0$. In order to define an upper bound for $\varepsilon$, define $Q:=A^{\top} \tilde{\Psi}+\tilde{\Psi} A$. Since $\tilde{\Psi}$ is symmetric and positive definite, there exists a nonsingular matrix $N \in \mathbb{R}^{n_{1} \times n_{1}}$ such that $\tilde{\Psi}:=N^{\top} N$. Consequently, $\varepsilon$ is such that $Q+2 \varepsilon \tilde{\Psi}<0$ if and only if $N^{-\top} Q N^{-1}+2 \varepsilon<0$. In order for this to hold, $\varepsilon$ must be less than $-\frac{1}{2} \lambda_{\max }$, where $\lambda_{\max }$ is the largest eigenvalue of $N^{-\top} Q N^{-1}$. Consequently, $\varepsilon$ must necessarily belong to the interval $\left[0,-\frac{1}{2} \lambda_{\max }\right)$.

Based on this discussion, we state the following algorithm.

\section{Algorithm 1:}

Input: Hurwitz polynomials $p_{1}, p_{2}$ with $n_{1}>n_{2}+1$. Output: If they exist, two strictly- positive-real completions.
Step 1: Define $A, B$ as in the controllable canonical realization of $\frac{1}{p_{1}}$, and $C^{\top}:=\tilde{T} \tilde{m}$ as in (3).

Step 2: Solve the LMI (4) with $\varepsilon=0$, to obtain $\tilde{\Psi}_{0}$ and the coefficient vector $\tilde{m}_{0}$. If there is no solution, EXIT.

Step 3: Compute a factorization $\tilde{\Psi}_{0}:=N_{0}^{\top} N_{0}$ and define $Q_{0}:=A^{\top} \tilde{\Psi}_{0}+\tilde{\Psi}_{0} A$.

Step 5: Compute the largest eigenvalue $\lambda_{\max , 0}$ of $N_{0}^{-\top} Q_{0} N_{0}^{-1}$, and choose $\varepsilon_{1} \in\left(0,-\frac{1}{2} \lambda_{\max , 0}\right)$.

Step 2: Solve the LMI (4) with $\varepsilon=\varepsilon_{1}$, to obtain $\tilde{\Psi}_{1}$ and the coefficient vector $\tilde{m}_{1}$.

Step 6: RETURN $\tilde{m}_{0}$ and $\tilde{m}_{1}$.

\section{CONCLUSIONS}

We studied the stability of scalar switched linear differential systems with three behaviors using the concept of positive-real completion, and we illustrated how a family of switched differential systems can be obtained the convex combination of two completions.

\section{REFERENCES}

[1] B.D.O. Anderson and S. Vongpanitlerd. Network Analysis and Synthesis: A Modern Systems Theory Approach. Prentice-Hall, Inc., NJ, 1973.

[2] D. Liberzon. Switching in Systems and Control. Birkhauser. Boston, Basel, Berlin, 2003.

[3] H. Lin and P.J. Antsaklis. Stability and stabilizability of switched linear systems: A survey of recent results. IEEE Transactions on Automatic Control, 54(2):308-322, 2009.

[4] J.W. Polderman and J.C. Willems. Introduction to Mathematical System Theory: A Behavioral Approach. Springer, Berlin, 1997.

[5] P. Rapisarda and P. Rocha. Positive realness and Lyapunov functions. Proceedings of the 20th International Symposium on Mathematical Theory of Networks and Systems, 2012. Melbourne, Australia.

[6] P. Rocha, J.C. Willems, P. Rapisarda, and D. Napp. On the stability of switched behavioral systems. 50th IEEE Conference on Decision and Control and European Control Conference (CDC-ECC), pages 1534-1538, 2011.

[7] R. Shorten, M. Corless, K. Wulff, Steffi Klinge, and R. Middleton. Quadratic stability and singular siso switching systems. Automatic Control, IEEE Transactions on, 54(11):2714-2718, 2009.

[8] R. Shorten, F. Wirth, O. Mason, K. Wulff, and C. King. Stability criteria for switched and hybrid systems. SIAM Review, 49(4):545592, 2007.

[9] Z. Sun and S.S. Ge. Switched Linear Systems: Control and Design. Springer-Verlag, New York, 2005.

[10] G. Tao and P.A. Ioannou. Necessary and sufficient conditions for strictly positive real matrices. In Circuits, Devices and Systems, IEE Proceedings G. IET., 137(5), 1990.

[11] S. Trenn. Switched differential algebraic equations. Dynamics and Control of Switched Electronic Systems. Chapter 6 of: Francesco Vasca and Luigi Iannelli (eds.), Springer Verlag, 2012.

[12] J.C. Willems and H.L. Trentelman. On quadratic differential forms. SIAM J. Control Optim., 36:1703-1749, 1998.

[13] O. Wing. Classical Circuit Theory. Springer-Verlag, New-York, 2008.

[14] Ezra Zeheb, Robert Shorten, and S. Shravan K. Sajja. Strict positive realness of descriptor systems in state space. International Journal of Control, 83(9):1799-1809, 2010. 